\title{
VARIABILIDADE ESTACIONAL DO BALANCCO DE ONDAS LONGAS EM UM ECOSSISTEMA DE MATA ATLÂNTICA NO ESTADO DE ALAGOAS-BRASIL
}

\author{
Misael Ferreira dos Santos \\ Universidade Federal de Alagoas, Instituto de Ciências Atmosféricas, \\ Maceió, AL, Brasil \\ santos.msl.fr@gmail.com \\ Carlos Alexandre Santos Querino \\ Universidade Federal do Amazonas, Instituto de Educação Agricultura e Ambiente, \\ Humaitá, AM, Brasil \\ carlosquerino@ufam.edu.br \\ Jeová Ramos da Silva Junior \\ Universidade de São Paulo, Instituto de Astronomia, Geofísica e Ciências Atmosféricas, \\ São Paulo, SP, Brasil \\ jeova.junior@icat.ufal.br \\ Rayonil Gomes Carneiro \\ Instituto Nacional de Pesquisas Espaciais, Centro de Ciências do Sistema Terrestre, \\ São José dos Campos, SP, Brasil \\ rayonilcarneiro@gmail.com \\ José Marcelo Lopes Júnior \\ Instituto Nacional de Pesquisas Espaciais, Centro de Previsão de \\ Tempo e Estudos Climáticos, São José dos Campos, SP, Brasil \\ josemarcelolopesjunior@hotmail.com

Marcos Antonio Lima Moura
Universidade Federal de Alagoas, Instituto de Ciências Atmosféricas,
Maceió, AL, Brasil
malm@ccen.ufal.br

\begin{abstract}
RESUMO
O conhecimento do balanço de radiação ondas longas (BOL) é importante para determinação das trocas energéticas que ocorrem no sistema superfície-atmosfera. Assim, neste trabalho avaliou-se a variabilidade estacional do BOL numa área de Mata Atlântica, através da análise da influência de diferentes variáveis meteorológicas, discutindo suas possíveis causas. A pesquisa foi realizada numa Reserva Particular do Patrimônio Natural (RPPN) no município de Coruripe-AL. As medições foram feitas por um saldo radiômetro (CNR1, Kipp e Zonen) instalado acima do dossel florestal numa torre micrometeorológica de 26 metros de altura durante os períodos de 03.11.2016 a 31.12.2016 (estação seca) e 01.05.2017 a 30.06.2017 (estação chuvosa). O BOL variou em 101,63\% da estação chuvosa para a seca $\left(-11,6 \mathrm{~W}\right.$ m-2 a $\left.-23,39 \mathrm{~W} \cdot \mathrm{m}^{-2}\right)$ em dias nublados e $30,33 \%(-49,19$ $\mathrm{W} \cdot \mathrm{m}^{-2}$ a $-64,11 \mathrm{~W} \cdot \mathrm{m}^{-2}$ ) em dias claros. A radiação de onda longa atmosférica $(\mathrm{Ra})$ média no período chuvoso foi $430,96 \mathrm{~W} \cdot \mathrm{m}^{-2}$ e $418,90 \mathrm{~W} \cdot \mathrm{m}^{-2}$ no seco, enquanto a radiação terrestre (Rs) foi $456,46 \mathrm{~W} . \mathrm{m}^{-2}$ (chuvoso) e 470,60 W. $\mathrm{m}^{-2}$ (seco). Rs foi sempre maior que Ra e, por consequência, o BOL foi sempre negativo em ambas estações, com uma diferença média de 103,19\% de uma estação para outra.
\end{abstract}

Palavras-chave: Interação Biosfera-Atmosfera. Balanço de Radiação. Floresta.

\section{SEASONAL VARIABILITY OF THE LONGWAVE BALANCE IN THE MATA ATLÂNTICA ECOSYSTEM IN ALAGOAS STATE - BRAZIL}

\begin{abstract}
Knowledge of the net longwave radiation (BOL) is important for determining the energy exchange that occur in surface-atmosphere system. Thus, this work evaluated the seasonal
\end{abstract}


variability of $\mathrm{BOL}$ in an area of Atlantic Forest, through the analysis of the influence of different meteorological variables, discussing their possible causes. The research was performed in the Private Reserve of the Natural Patrimony (RPPN), county of Coruripe-AL. The measurements were made by a net radiometer (CNR1, Kipp and Zone) installed above the forest canopy in a 26 meter high micrometeorological tower during the periods from 03.11.2016 to 31.12.2016 (dry season) and 01.05.2017 to 30.06.2017 (rainy season). The BOL ranged from $101.63 \%$ from rainy to dry season $\left(-11.6 \mathrm{~W} \cdot \mathrm{m}^{-2}\right.$ to $\left.-23.39 \mathrm{~W} \cdot \mathrm{m}^{-2}\right)$ on cloudy days and $30.33 \%\left(-49.19 \mathrm{~W} \cdot \mathrm{m}^{-2}\right.$ to $\left.-64.11 \mathrm{~W} \cdot \mathrm{m}^{-2}\right)$ on clear days. The average of atmospheric longwave radiation $(\mathrm{Ra})$ in the rainy season was $430.96 \mathrm{~W} . \mathrm{m}^{-2}$ and $418.90 \mathrm{~W} \cdot \mathrm{m}^{-2}$ in the dry season while terrestrial radiation (Rs) was $456.46 \mathrm{~W} . \mathrm{m}^{-2}$ (rainy) and $470.60 \mathrm{~W} . \mathrm{m}^{-2}$ (dry). Rs was always higher than $\mathrm{Ra}$, and therefore the $\mathrm{BOL}$ was always negative in both seasons, with an average difference of $103.19 \%$ from one season to another.

Keywords: Biosphere-Atmosphere Interaction. Radiation Balance. Forest.

\section{INTRODUÇÃO}

A Mata Atlântica é oriunda de um conjunto de formações florestais (Ombrófila Densa, Mista, Estacional Semidecidual, Estacional Decidual e Aberta), ecossistemas de restingas, manguezais e campos de altitude (MMA, 2010). Originalmente se estendia por aproximadamente $1.300 .000 \mathrm{~km} 2 \mathrm{em}$ 17 estados do território brasileiro. Representa para o planeta $0,8 \%$ da superfície terrestre, mais de $5 \%$ dos vertebrados e cerca de $5 \%$ da sua flora. Estima-se que mais de 15.700 espécies estão presentes no bioma. Foi decretada como Reserva da Biosfera pela Unesco e Patrimônio Nacional, na Constituição Federal de 1988 e dessa forma passou a ser considerada um Hotspot mundial (SOS Mata Atlântica/INPE, 2018) porém, ainda segundo o referido artigo, tem-se apenas $8,5 \%$ de remanescentes florestais, com área acima de 100 hectares.

As práticas antrópicas estão ligadas diretamente ao desmatamento de sistemas florestais para transformação em sistemas agrícolas e/ou pastagem (NOBRE et al., 2007). Como consequência deste desmatamento, há uma maior transferência de carbono da biosfera para a atmosfera o que gera, consequentemente, mudanças climáticas. Além do impacto quanto à emissão de carbono para a atmosfera, o desmatamento influencia diretamente o microclima, devido às alterações nos balanços de radiação de ondas curtas e longas (QUERINO et al., 2017). Alterações nos fluxos radiativos, devido a substituição da floresta por pastagem, implica na redução da evapotranspiração durante o dia (LYRA et al., 1994). Essa redução na evapotranspiração implica em diminuição no fluxo energético destinado à calor latente e aumento no fluxo de calor sensível e, por conseguinte, aumento da temperatura da superfície (BIUDES et al., 2015). Essas modificações na temperatura da superfície acarretam, dentre outros fatores, em alterações no fluxo de ondas longas superficiais.

O balanço de radiação à superfície é importante para entender o sistema climático e sua variabilidade (PASHIARDIS et al., 2017). As radiações de ondas curtas (OC) e longas (OL) ascendentes (Rs) e descendentes (Ra) são os fluxos envolvidos nos processos de trocas de energia no sistema terraatmosfera. A troca de OL é um processo bidirecional. A Ra é absorvida pela superfície e simultaneamente enviada para atmosfera e depende das várias camadas gasosas na atmosfera que são aquecidas a uma gama de temperaturas. Já a Rs depende das propriedades de emissão/absorção do material à superfície e de sua temperatura.

Nos últimos anos, estudos (PRATA, 1996; GALVÃO e FISCH, 2000; SOUZA-FILHO et al., 2006; AGUIAR, 2007; QUERINO, 2016) abordaram o problema de estimar OL a partir de variáveis atmosféricas simples e amplamente disponíveis (ex.: temperatura e umidade relativa do ar). Existem várias maneiras de estimar as componentes da radiação de OL (ex.: modelos transferência radiativa ou medições diretas por pirgeômetros). 
Medição direta é o método mais preciso, mas medidas regulares de Ra e Rs à superfície são poucas. Ainda são escassos os estudos microclimáticos, principalmente relacionados a OL, sobre Floresta de Mata Atlântica (CARNEIRO et al., 2013). Desta forma, evidencia-se, que não há registros de séries de dados similares, bem como nenhuma análise símile para região de Mata Atlântica em fonte indexada.

Em decorrência do exposto, o objetivo desse trabalho foi caracterizar a variabilidade sazonal e a influência de variáveis micrometeorológicas no balanço de ondas longas (BOL) através de medições em um fragmento remanescente de floresta de Mata Atlântica.

\section{METODOLOGIA}

\section{ÁREA DE ESTUDO}

A pesquisa foi realizada em um fragmento de Floresta Ombrófila Aberta em transição para Floresta Estacional Semidecidual inserido na região dos Tabuleiros Costeiros com uma área de 68,6 ha (MACHADO, 2003) localizadas dentro da Reserva Particular do Patrimônio Natural (RPPN) - Lula Lobo I, no município de Coruripe, Alagoas (Figura 1).

O clima da região, segundo a classificação de Köppen, é do tipo As', ou seja, tropical com período chuvoso entre março e agosto e período seco de setembro a fevereiro com total pluviométrico médio anual de $1.800 \mathrm{~mm}$. Segundo Ferreira Junior et al. (2014) as médias climatológicas anuais da temperatura, umidade relativa do ar e fração de nebulosidade são $25,4^{\circ} \mathrm{C}, 81,8 \%$ e $42,9 \%$, respectivamente. $O$ vento apresenta direções predominantes de nordeste (outubro a março) e sudeste (abril a setembro) (MACHADO, 2003). A irradiação solar global média mensal varia de 14,8 MJ.m-2 em julho a 24 MJ.m-2 em novembro (SOUZA et al., 2005).

Figura 1 - Localização da torre micrometeorológicas utilizada no experimento.

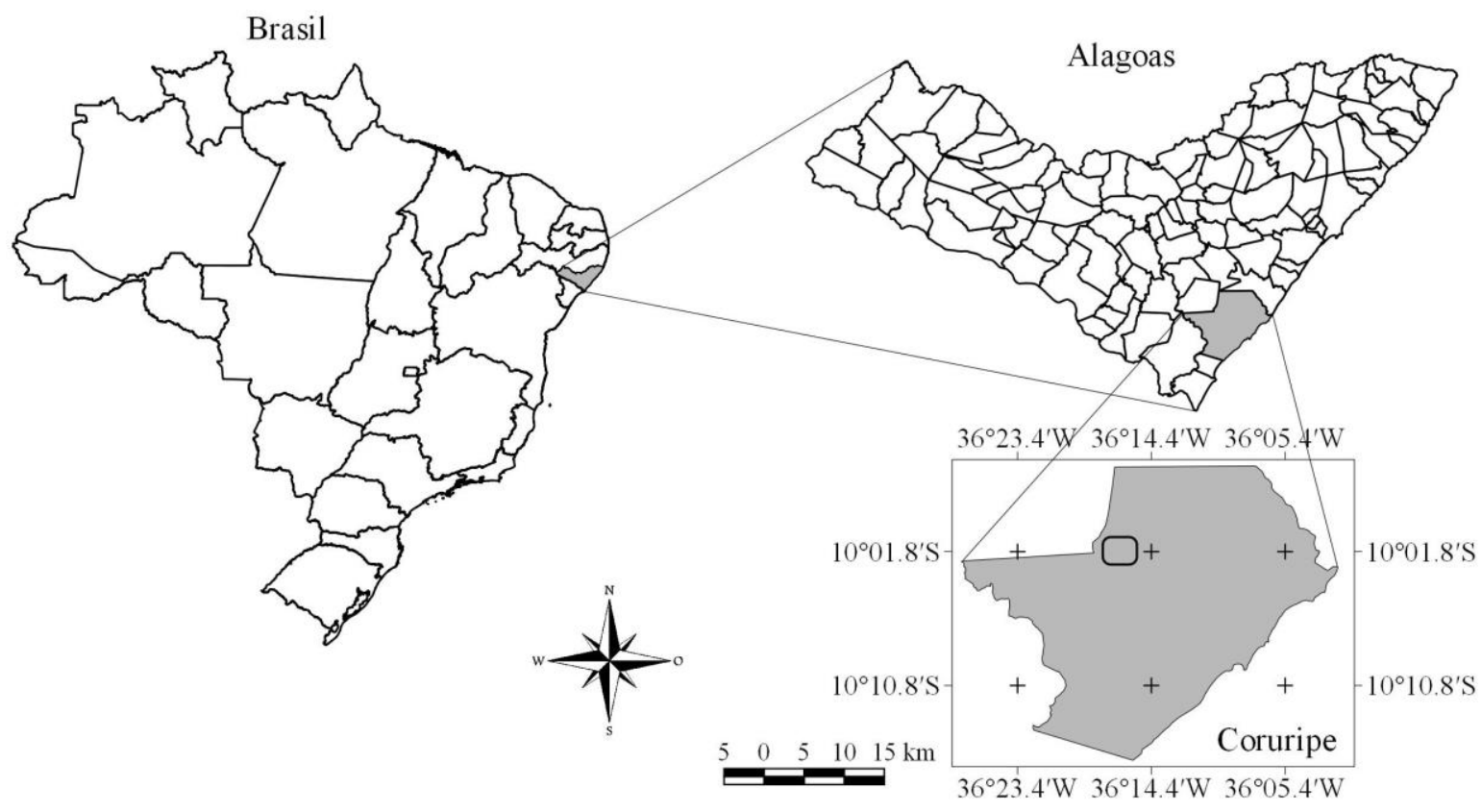

\section{ANÁLISE E COLETA DE DADOS}

Os dados foram analisados para no período de 03/11/2016 a 31/12/2016 (estação seca) e de 01/05/2017 a 30/06/2017 (estação chuvosa). Os registros foram realizados a cada 20 segundos por

$\begin{array}{lllll}\text { Caminhos de Geografia } & \text { Uberlândia-MG } & \text { v. 21, n. } 75 & \text { Jun/2020 } & \text { p. 201-213 Página } 203\end{array}$


equipamentos instalados acima da copa das árvores, em uma torre micrometeorológica (1000'37"S, $36^{\circ} 17$ '60"W, $160 \mathrm{~m}$ alt.) de $26 \mathrm{~m}$ de altura e as médias armazenadas a cada 10 minutos em dois dataloggers (CR10X, Campbell Scientific). As medições da radiação de onda longa atmosférica (Ra), terrestre (Rs), radiação solar global incidente $(\mathrm{Rg})$ e refletida foram realizadas por um saldo radiômetro modelo CNR1 da Kipp e Zonen (Delft, Holanda). A precipitação foi registrada pelo pluviômetro TE 525 (Texas Instruments, USA). Também foram utilizadas as normais climatológicas (NC) do município de Coruripe, Alagoas, Brasil, disponibilizadas pelo Instituto Nacional de Meteorologia (INMET).

\section{ESTIMATIVA DO ÍNDICE DE TRANSMISSIVIDADE DA ATMOSFERA (KT)}

O índice de transmissividade da atmosfera $(\mathrm{Kt})$ foi calculado a partir da razão entre a radiação solar incidente na superfície terrestre $(\mathrm{Rg})$ e a radiação solar incidente no topo da atamosfera (Ro) (Equação 1).

$\mathrm{Kt}=\frac{\mathrm{Rg}}{\mathrm{Ro}}$

(1)

O Ro foi estimado de acordo com IQBAL (1983) (Equação 2), no qual considerou que Ro é determinado em função da Latitude do local $(\varphi)$, da Constante Solar $\left(S o=1.367\right.$ W.m $\left.{ }^{-2}\right)$, da Correção da Excentricidade do Raio Vetor da Órbita da Terra (Eo) (Equação 3), do Cosseno do Ângulo Zenital (z) (Equação 4), que por sua vez depende da Declinação Solar (ס) (Equação 5) e do Ângulo Horário (H) (Equação 6).

$R o=$ So. Eo. $\cos \mathrm{Z}$

onde:

Eo $=1+0,0033 \cdot \cos \left(\frac{2 \pi d n}{365}\right)$

em que dn é o número do dia no calendário Juliano. Já o ângulo zenital solar (Z) é dado pela equação:

$\cos Z=\operatorname{sen} \varphi \cdot \operatorname{sen} \delta+\cos \varphi \cdot \cos \delta \cdot \cos \mathrm{H}$

sendo $\delta$ a declinação solar e H o ângulo horário, calculados com as respectivas expressões:

$\delta=23,45 . \operatorname{sen}\left[\frac{360}{365}(284+d n)\right]$

$\mathrm{H}=\arccos (-\operatorname{tg} \varphi \cdot \operatorname{tg} \delta)$

Foram adotados os intervalos de referência da transmitância atmosférica para a região de Maceió-AL de $\mathrm{Kt} \geq 0,6$ (condições de céu claro $(\mathrm{CL})$ ou com pouca nebulosidade), 0,4 $\leq \mathrm{Kt}<0,6$ (céu parcialmente nublado (PN) ou com média nebulosidade) e $\mathrm{Kt}<0,4$ (céu nublado (NB) ou com alta nebulosidade) (LOPES JUNIOR et al., 2018).

\section{RESULTADOS E DISCUSSÃO}

\section{ESTAÇÃO CHUVOSA}

A precipitação foi de 382,9 mm (maio) e 490,3 mm (junho). De acordo com a normal climatológica do município de Coruripe-AL, a precipitação total para os mesmos meses deve ser, respectivamente, para maio e junho de 190,4 e 223,8 mm. Essa grande quantidade de precipitação acima do previsto 
ocorreu devido as anomalias positivas de temperatura da superfície do mar, próximo à costa sudeste da América do Sul, por cinco meses consecutivos (SEMARH, 2017). Para o mesmo período, constatou-se que $72,13 \%$ das chuvas ocorreram em dois momentos com intensa precipitação diária, concentrados, principalmente, pela manhã: segunda quinzena de maio $(264 \mathrm{~mm})$ e de junho $(410$ $\mathrm{mm}$ ) (Figura 2a).

De acordo com Pai e Escobedo (2015) o índice Kt é um indicativo dos processos de absorção e espalhamento da radiação por nuvens, aerossóis e vapor d'água. É relatado na literatura por expressar a condição de nebulosidade do céu. Este índice é importante para o cálculo de Ondas Longas (OL) em qualquer condição de cobertura de céu, pois é um bom indicador da presença de água na atmosfera. Os valores de precipitações influenciaram no Kt que apresentou $65,57 \%$ de dias nublados (NB), 34,42\% parcialmente nublado (PN) e $0 \%$ céu limpo (CL), corroborando com os maiores valores de $\mathrm{Rg}$ na primeira quinzena de maio (figura 2b). Nota-se também grande variabilidade diária com características semelhantes nos períodos matutino e vespertino, diferente dos encontrados por Souza et al. (2005). Possivelmente, esta diferença está associada à variabilidade na cobertura de nuvens, uma vez que os autores encontraram Kt $(20 \%=N B ; 56 \%=P N$; $24 \% \mathrm{CL}$ ), os quais diferem muito e acarretaram esta diferença na $\mathrm{Rg}$.

Em condições de nebulosidade tem-se menos $\mathrm{Rg}$ incidente à superfície, menos energia é armazenada e, consequentemente, menor Rs. Percebe-se claramente que as maiores Rs concentram-se entre 6 e 18 horas (período com presença de luz solar), só que com grande variabilidade (figura 2d). Os maiores picos da Rs são observados após os horários de maior incidência da $\mathrm{Rg}$, já que as maiores temperaturas da superfície do dossel também são observadas nesses horários.

Segundo Souza-Filho et al. (2006) o vapor de água e $0 \mathrm{CO}_{2}$ são considerados como termorreguladores atmosféricos em razão da capacidade de absorver radiação de ondas longas. Assim, dias com maior frequência de precipitação estão associados com menor amplitude na Ra e, consequentemente, o período diurno assemelha-se ao noturno como por exemplo no dia 28 de maio, quando a Ra (Figura 2c) é praticamente homogênea. Durante o período de estudo, a máxima (mínima) observada na Ra foi de 481,27 W.m² (373,28 W.m²).

O BOL é controlado, basicamente, pelas temperaturas do ar e do solo, nebulosidade e concentrações de vapor d'água e dióxido de carbono na atmosfera. Valores de BOL $<0 \mathrm{~W} . \mathrm{m}^{-2}$ indicam que a superfície emite mais do que a atmosfera, pois sua temperatura é maior. Os menores BOLs ocorreram entre às 6 e $18 \mathrm{~h}$ (figura $2 \mathrm{e}$ ) e os maiores nos períodos noturnos e inícios das manhãs. Em dias de chuva o $\mathrm{BOL}$ foi próximo a zero durante todo o dia num claro equilíbrio das temperaturas do ar e superfície. Isso corrobora com Singh et al. (2014) sobre um estudo na Índia, na qual o BOL aumenta com a precipitação. O BOL variou de $-11,6 \mathrm{~W} . \mathrm{m}^{-2}$ em dias nublados para $-49,19 \mathrm{~W} \cdot \mathrm{m}^{-2} \mathrm{em}$ dias claros.

A Ra, em médias horárias, variou entre 404,85 W.m-2 e 447,17 W.m-2 (média de 431,04 W.m-2), enquanto a Rs entre 442 W.m. $\mathrm{m}^{-2}$ e $581 \mathrm{~W} \cdot \mathrm{m}^{-2}$ (média de 456,75 W.m²). Assim, Ra e Rs, para o período considerado, apresentaram-se bem definidas com curvas senoidais com máximos em torno do meio dia e mínimos próximos às 5 horas (Figura 3a). Na floresta amazônica, Aguiar (2007) encontrou 429 W.m. ${ }^{-2}$ (Ra), Souza-Filho et al. (2006) 417 W.m. ${ }^{-2}$ (Ra) e 339 W.m.- (Rs) e Bastable et al. (1993) 418 W.m-2 (Ra). Não obstante os resultados obtidos das variações horárias da Ra são muito complexos durante o dia. As nuvens são um fator importante na Ra porque absorvem e emitem fluxos para baixo e para cima. Sabe-se que a presença de nuvens modifica substancialmente o fluxo de radiação atmosférica que é recebido na superfície, porque a radiação emitida pelo vapor de água e dióxido de carbono na atmosfera inferior é suplementada pela emissão das nuvens (MALEK, 1997). 
Misael Ferreira dos Santos

Carlos Alexandre Santos Querino Jeová Ramos da Silva Junior

Rayonil Gomes Carneiro

Variabilidade estacional do balanço de ondas longas em um ecossistema de Mata Atlântica no estado de Alagoas-Brasil

José Marcelo Lopes Júnior Marcos Antonio Lima Moura

Figura 2 - Acumulados horários-diários de a) precipitação $(\mathrm{mm})$ e médias horárias-diárias (W.m-2) de b)

Radiação solar global $(\mathrm{Rg})$, c) radiação de onda longa atmosférica (Ra), d) radiação de onda longa emitida pela superfície (Rs) e) balanço de onda longa (BOL), na estação chuvosa.

a)

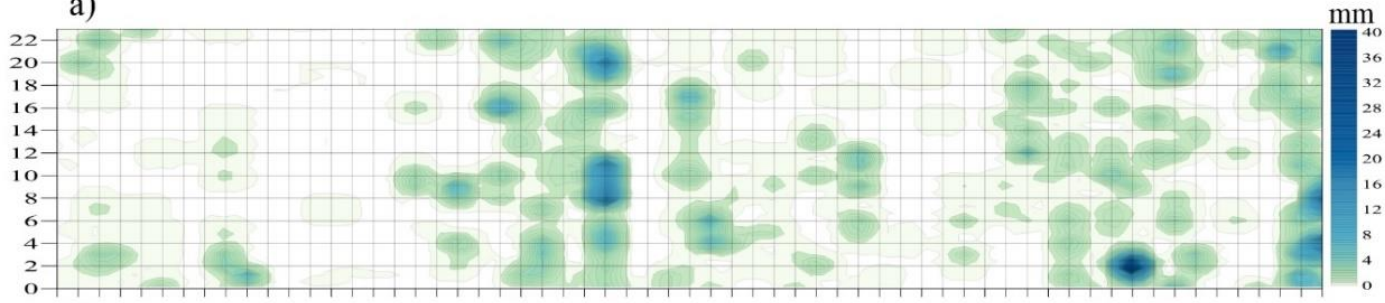

b)

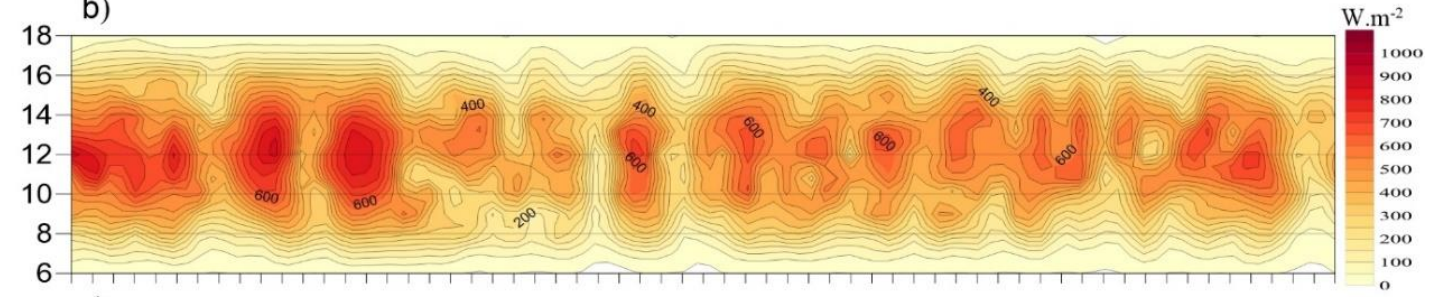

c)

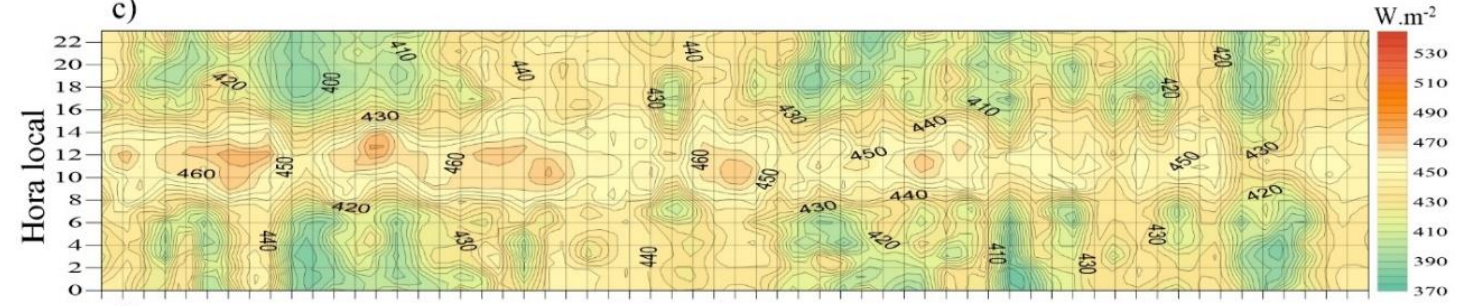

d)

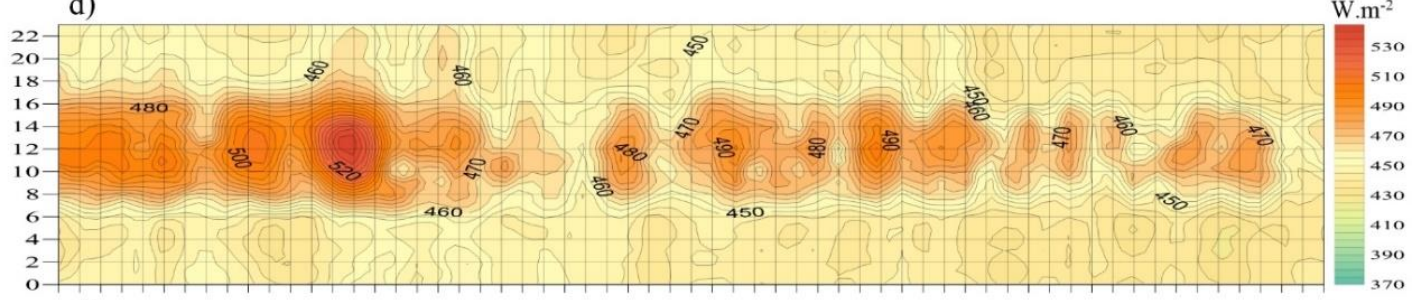

e)

W.m-2

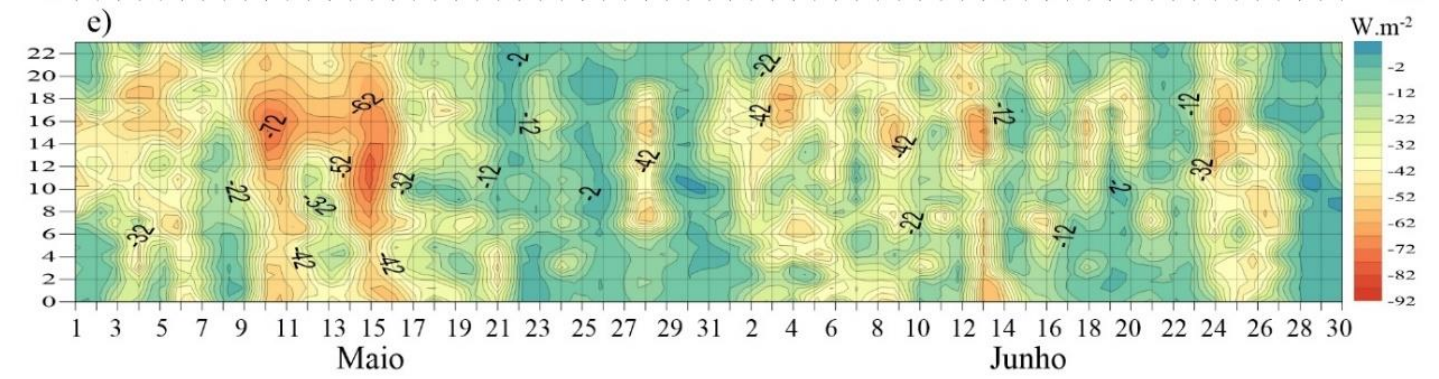

Verificou-se que o BOL se torna menor a partir das 9 horas e atinge seu máximo às 16 horas com média horária de $-33,82 \mathrm{~W} \cdot \mathrm{m}^{-2}$ e mantem-se constante durante todo período noturno. Presume-se que isso acontece pelo fato da temperatura do dossel atinge seu máximo neste horário. Já quando se analisa a distribuição de frequência (Figura $3 b$ e $3 c$ ) verifica-se que a Ra apresenta uma assimetria negativa (cauda à esquerda) com Mediana de 435,62 W. $\mathrm{m}^{-2}$. Em contrapartida, a Rs apresenta uma assimetria positiva (cauda à direita) e Mediana de $451,38 \mathrm{~W} \cdot \mathrm{m}^{-2}$.

Segundo Oke (1987), em noites de céu nublado a Ra pode ser superior à Rs, devido aos processos de reflexão e emissão ocasionados pela presença de nuvens, mas isso não foi verificado no estudo em tela. Galvão e Fisch (2000), esperavam obter valores superiores de Ra em relação à Rs na região amazônica em dias nublados, já que as nuvens são as maiores contribuidoras de Ra para a superfície, pois irradiam como corpo negro a partir da base das nuvens, mas também não foi o observado. Devido a essa dinâmica as médias diárias do BOL também apresentaram variações em dias com precipitação, então o BOL oscilou entre -65,4 W. $\mathrm{m}^{-2}$ e $-0,94 \mathrm{~W} \cdot \mathrm{m}^{-2}$ (Figura 4).

$\begin{array}{lllll}\text { Caminhos de Geografia } & \text { Uberlândia-MG } & \text { v. 21, n. } 75 & \text { Jun/2020 } & \text { p. 201-213 Página } 206\end{array}$ 
Misael Ferreira dos Santos

Carlos Alexandre Santos Querino Jeová Ramos da Silva Junior

Rayonil Gomes Carneiro

Variabilidade estacional do balanço de ondas longas em um ecossistema de Mata Atlântica no estado de Alagoas-Brasil

José Marcelo Lopes Júnior Marcos Antonio Lima Moura

Figura 3 - a) Variação média horária da irradiância atmosférica $(\mathrm{Ra})$, superficial (Rs) e do balanço de ondas longas (BOL), todos em W.m-2; b) distribuição da frequência da irradiância atmosférica (Ra) e c) superficial (Rs) na estação chuvosa.

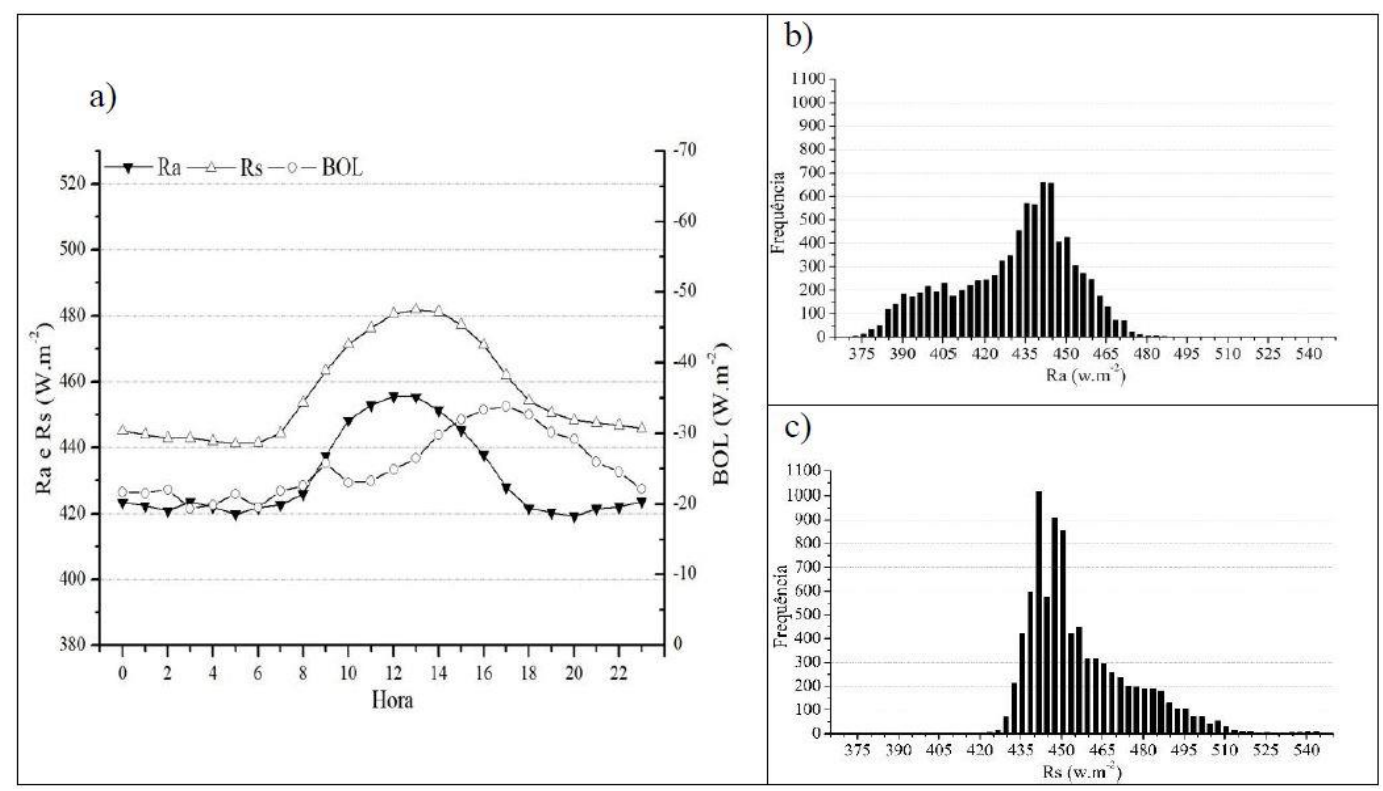

Vale ressaltar que a nebulosidade desempenha um papel significativo na quantidade da irradiância descendente emitida, mas a quantidade de chuva poderá sim influenciar as Ra, Rs e BOL, pois pode estar relacionada com chuvas intensas e/ou duradouras ao longo do dia. Constatou-se que não somente em dias com maiores registros de precipitação a Ra e Rs tendem a serem próximas (Figura 4), o que acarreta em BOL próximo de zero. Para que isso ocorra, dois fenômenos atuam simultaneamente: primeiramente, resfriamento intenso da superfície do dossel e, consequente, diminuição da Rs; segundo, maior contribuição da (re) emissão por parte das nuvens na Ra. Este fato é mais evidente no final de maio e junho, respectivamente. Isso pode ser melhor exemplificado nos dias: 30.05 com registro de $4,3 \mathrm{~mm}$, kt de 0,15 e BOL igual $-2,24 \mathrm{~W} \cdot \mathrm{m}^{-2}$, enquanto $29.06 \mathrm{com} 71 \mathrm{~mm}$, 0,17 de kt e BOL de $-0,94$ W.m-2.

Figura 4 - Média diária da radiação de onda longa emitida pela atmosfera (Ra), pela superfície (Rs), do balanço de onda longa (BOL), em W.m-2, e acumulado diária de precipitação (Precip), em mm, na estação chuvosa.

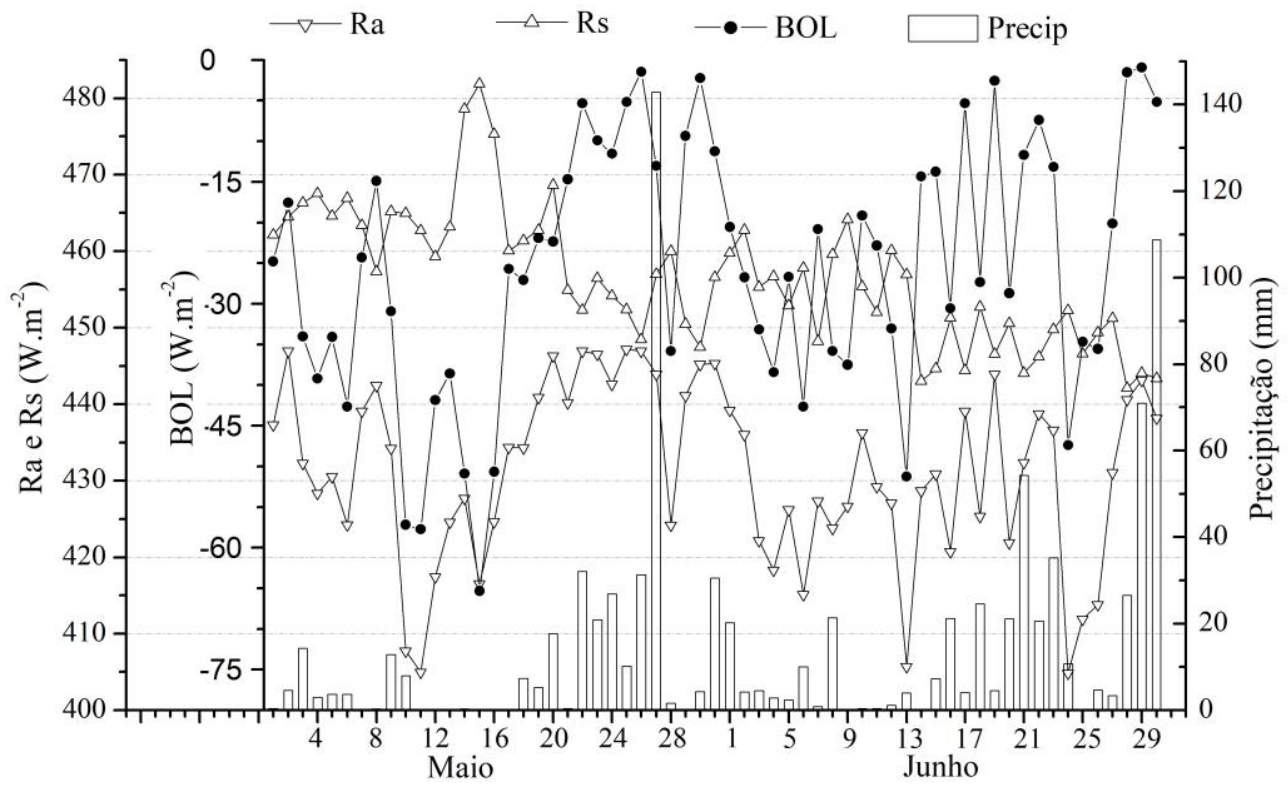




\section{ESTAÇÃO SECA}

A precipitação total foi de 2,8 $\mathrm{mm}$ (novembro) e 15,3 $\mathrm{mm}$ (dezembro) correspondendo a 20 e $60 \%$ do esperado, respectivamente (Figura 5a). Segundo a SEMARH (2017), os campos sinóticos apresentaram padrões comumente associados a anos de seca sobre a porção norte do Nordeste. Somente $12 \%$ dos dias teve ocorrência de chuva, mas apenas um dia (20/12/2016) de chuva significativa $(\sim 10 \mathrm{~mm})$.

Esta situação refletiu no Kt, que apresentou 5,66\% NB, 47,17\% PN e 47,17\% CL. O Kt é um indicador geral dos processos de absorção e espalhamento da radiação, por nuvens, aerossóis, vapor d'água, que intervêm na transmissão da radiação solar global através da atmosfera. As variações horárias do Rg (Figura 5b) são semelhantes às encontradas por Querino et al. (2011) e Souza et al. (2005), em torno de 900 W.m-2. Também coincidiu com Querino et al. (2011) o fato do Rg não apresentar simetria diurna e com crescimento mais acentuado no período matutino devido à um Kt maior pela manhã do que a tarde. O motivo para essa redução, na parte da tarde, pode ser atribuído a uma maior concentração de aerossóis marinhos trazidos por ventos de Leste, que segundo Santos (2006), são mais intensos entre 10 e $14 \mathrm{~h}$, os quais ao chegar à costa convergem para níveis superiores intensificando a formação de nuvens a tarde.

A nebulosidade desempenha um papel significativo na quantidade da irradiância descendente emitida. A Ra segue um padrão com máximos por volta do meio dia e mínimos às 20 horas (Figura 5c) mas alguns dias foram descaracterizados deste padrão em razão das condições atmosféricas presentes. A maior média horária de Ra foi de $477,65 \mathrm{~W} \cdot \mathrm{m}^{-2}$ e a menor de $370,51 \mathrm{~W} \cdot \mathrm{m}^{-2}$.

Maiores Rs (Figura 5d) estão compreendidas entre $6 \mathrm{~h}$ e $17 \mathrm{~h}$, uma vez que a mesma tende a ser maior devido maior Rg na estação, enquanto a maior média horaria foi $543,05 \mathrm{~W} \cdot \mathrm{m}^{-2}$ e menor 426,08 W. $\mathrm{m}^{-2}$. Como teve maior incidência da $\mathrm{Rg}$, logo ocorreu um maior fluxo de energia e aumento da temperatura. Entretanto, segundo Pezzopane et al. (2002), o dossel da floresta funciona também como um anteparo, impedindo trocas intensas de energia. Pires et al. (2015) relata que nos períodos mais quentes do dia, as folhas sempre expostas à radiação solar direta se encontravam mais quentes do que a temperatura ambiente, pois o déficit hídrico causa maior fechamento estomático, com consequente aumento da temperatura da folha, uma vez que não há dissipação suficiente de calor por meio do processo transpiratório.

Como a Rs é normalmente maior que Ra, então o BOL é negativo. Logo, quanto menor o BOL, mais energia é emitida (perdida) pela superfície. O BOL, em média, correspondeu a $10 \%$ do saldo de radiação total no período diurno, sendo a porcentagem maior nos inicios das manhãs e fins de tardes. O BOL mostrou grande variabilidade mas nota-se que, de modo geral, os menores valores horários ocorrem entre $10 \mathrm{~h}$ e 18h, enquanto os maiores no início da manhã (Figura 5e). A menor média horária foi $-91,49 \mathrm{~W} . \mathrm{m}^{-2}$ e a maior $-3,93 \mathrm{~W} . \mathrm{m}^{-2}$. Em média, o BOL variou de $-23,39 \mathrm{~W} . \mathrm{m}^{-2}$ em dias nublados para $-64,11 \mathrm{~W} \cdot \mathrm{m}^{-2} \mathrm{em}$ dias claros.

As curvas de Ra e Rs seguem um padrão visualmente simétrico (Figura 6a), sendo que a Rs apresenta seu máximo $\left(514,49 \mathrm{~W} \cdot \mathrm{m}^{-2}\right)$ às 13 horas e mínimo às 5 horas $\left(441,68 \mathrm{~W} \cdot \mathrm{m}^{-2}\right)$. Souza-Filho et al. (2006) encontraram na floresta amazônica $431 \mathrm{~W} \cdot \mathrm{m}^{-2}$ e Aguiar (2007) $435 \mathrm{~W} \cdot \mathrm{m}^{-2}$. Já a Ra exibe mínimo $\left(397,18 \mathrm{~W} \cdot \mathrm{m}^{-2}\right)$ com variação de somente $4,73 \mathrm{~W} \cdot \mathrm{m}^{-2} \mathrm{em}$ todo período noturno ao passo que seu máximo ocorreu às 12 horas local $\left(462,84 \mathrm{~W} . \mathrm{m}^{-2}\right)$. Aguiar (2007) encontrou média horária na floresta amazônica de $383 \mathrm{~W} \cdot \mathrm{m}^{-2}$, enquanto Souza-Filho et al. (2006) $433 \mathrm{~W} \cdot \mathrm{m}^{-2}$ e Bastable et al. (1993) 411 W. $\mathrm{m}^{-2}$. O balanço de radiação de ondas longas apresentou uma amplitude diária média de $-21,80 \mathrm{~W} \mathrm{~m}^{-2}$, porém a magnitude absoluta dos seus fluxos é maior em torno das 15 horas. 
Misael Ferreira dos Santos Carlos Alexandre Santos Querino Jeová Ramos da Silva Junior

Rayonil Gomes Carneiro

Variabilidade estacional do balanço de ondas longas em um ecossistema de Mata Atlântica no estado de Alagoas-Brasil

José Marcelo Lopes Júnior Marcos Antonio Lima Moura

Figura 5 - Acumulados horários-diários de a) precipitação $(\mathrm{mm})$ e médias horárias-diárias (W.m-2) de b)

Radiação solar global $(\mathrm{Rg})$, c) radiação de onda longa atmosférica (Ra), d) radiação de onda longa emitida pela superfície (Rs) e) balanço de onda longa (BOL), na estação seca.

a)
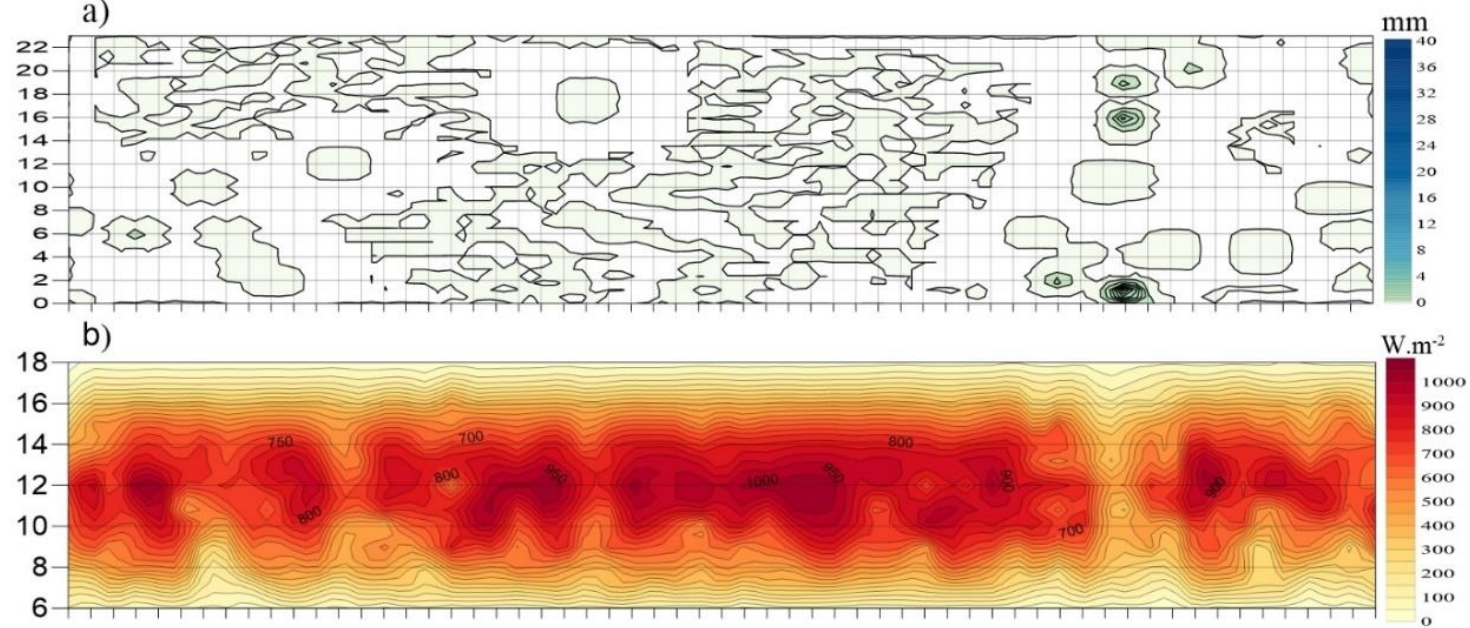

c)

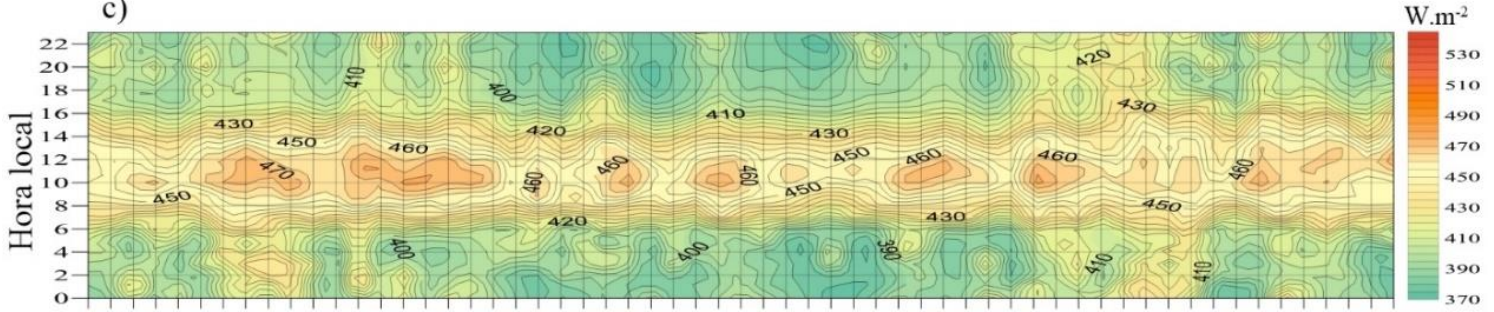

d)

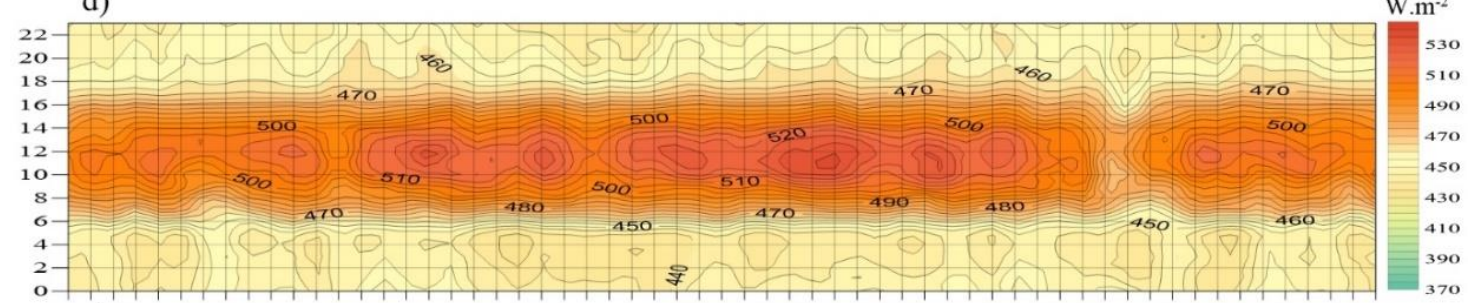

e)

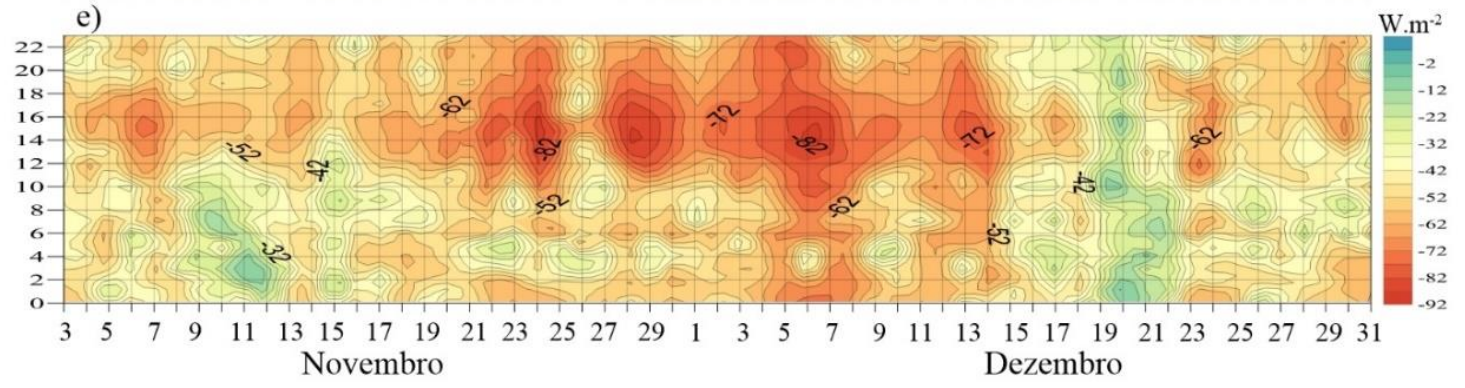

$\mathrm{O}$ BOL variou de $-42,14 \mathrm{~W} \mathrm{~m}^{-2}$ às $\sim 6$ horas a $-63,94 \mathrm{~W} \mathrm{~m}^{-2}$ às 15 horas devido a temperatura da superfície do dossel ser menor às 6 horas e maior às 16 horas. Nota-se que o BOL, em termos de valores, tem uma certa dependência da Rs, apesar de que sua curva ser visualmente parecida com a da Ra. Observou-se assimetria da distribuição de frequência para Ra e Rs (Figura 6 a e b), uma vez que ocorreram pouquíssimas variações em relação à nebulosidade e precipitação. Ambos, Ra e Rs, apresentam assimetria positiva e mediana de $414,30 \mathrm{~W} \cdot \mathrm{m}^{-2}$ (Ra) e 460,78 W.m ${ }^{-2}$ (Rs). Sabe-se que em dias nublados/precipitação o BOL é praticamente nulo, mas isso não foi constatado neste período, justamente pela quase total ausência de ambos (Figura 7) e, consequente maior amplitude entre Ra e Rs, uma vez que Ra variou entre $402,7 \mathrm{~W} \cdot \mathrm{m}^{-2}$ e $438,3 \mathrm{~W} \cdot \mathrm{m}^{-2}$, enquanto Rs entre $455,32 \mathrm{~W} \cdot \mathrm{m}^{-2} \mathrm{e}$ $487,6 \mathrm{~W} \cdot \mathrm{m}^{-2}$. 
Misael Ferreira dos Santos Carlos Alexandre Santos Querino Jeová Ramos da Silva Junior

Rayonil Gomes Carneiro

Variabilidade estacional do balanço de ondas longas em um ecossistema de Mata Atlântica no estado de Alagoas-Brasil

José Marcelo Lopes Júnior Marcos Antonio Lima Moura

Figura 6 - a) Variação média horária da irradiância atmosférica (Ra), superficial (Rs) e do balanço de ondas longas (BOL), todos em W.m-2; b) distribuição da frequência da irradiância atmosférica (Ra) e c) superficial (Rs) na estação seca.

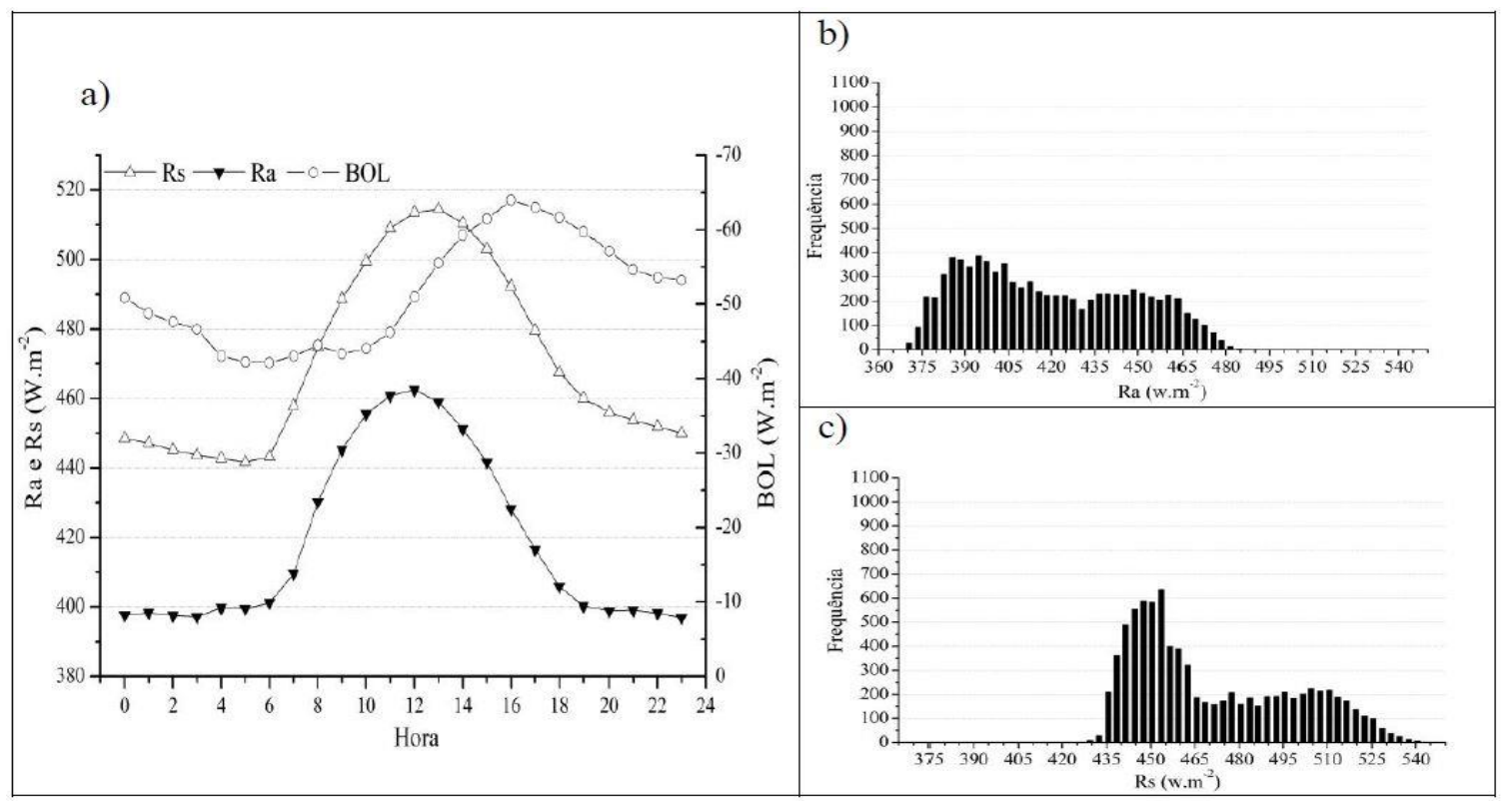

Figura 7 - Média diária da radiação da onda longa emitida pela atmosfera (Ra), pela superfície (Rs), do balanço de onda longa (BOL), em W.m-2, e acumulado diário de precipitação (Precip.), em mm, na estação seca.

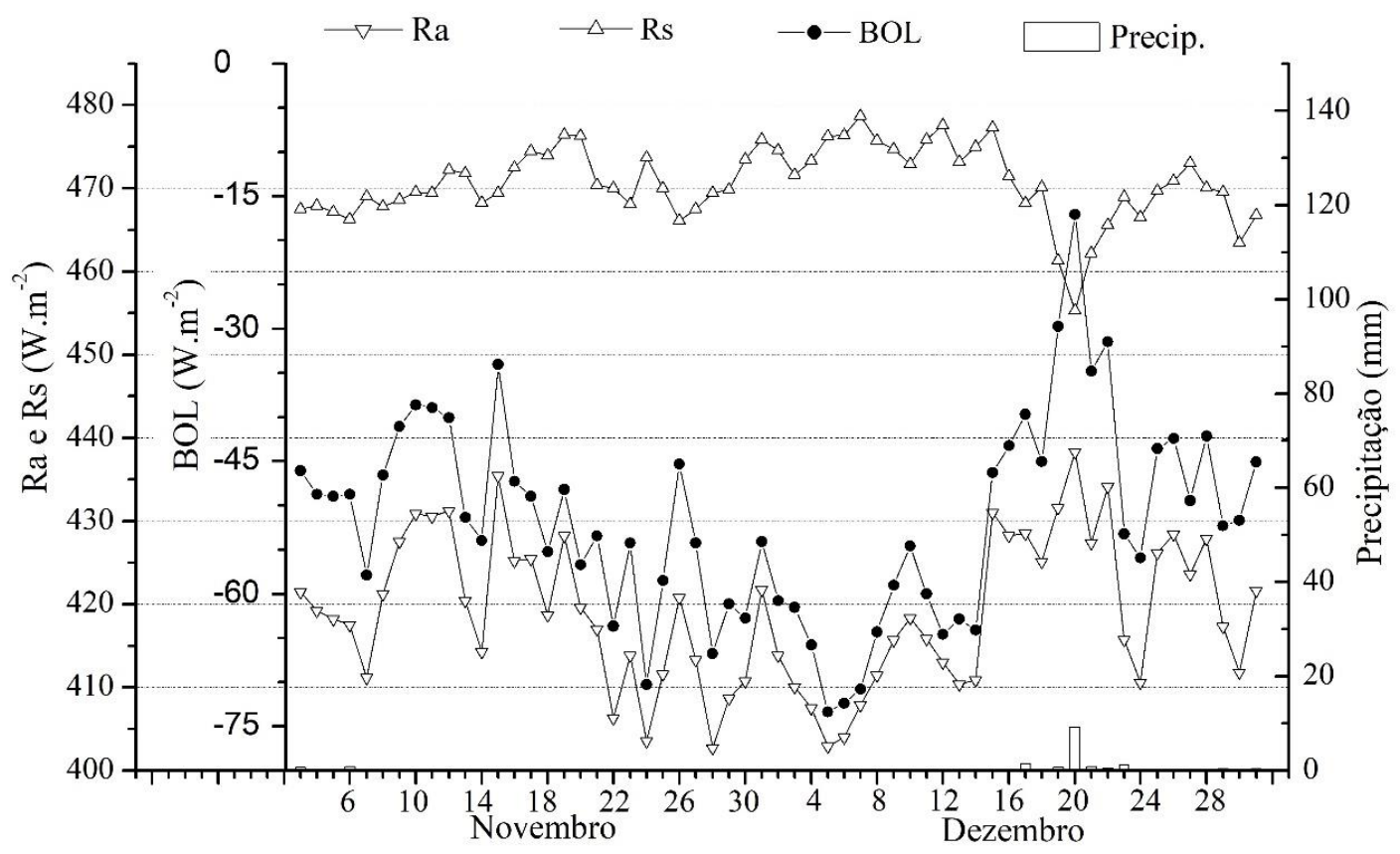

\section{CONSIDERAÇÕES FINAIS}

Balanço de ondas longas (BOL) e suas componentes variaram substancialmente com a dinâmica das nuvens, precipitação, período seco e chuvoso e condições hídricas da floresta, uma vez que os períodos analisados foram muito acima ou abaixo da média, já que choveu $52,56 \%$ acima da média no chuvoso e $53,8 \%$ abaixo da média no período seco. Dessa forma, ressalta-se a importância da temperatura da superfície do dossel e da nebulosidade nessas oscilações, uma vez que o BOL oscilou, em média, da 
estação chuvosa para seca 101,63\% (de -11,6 W.m.-2 para -23,39 W.m-2) em dias nublados e $30,33 \%$ (de 49,19 W.m-2 para $-64,11$ W.m-2) em dias claros.

Sob condições nubladas/precipitação, a Ra aumentou $3 \%$ em relação ao seu valor sob condições de céu claro, mas não supera a Rs, em ambas as estações. Então, a média no período chuvoso de Ra foi de $430,96 \mathrm{~W} \cdot \mathrm{m}^{-2}$ e de $418,90 \mathrm{~W} \cdot \mathrm{m}^{-2}$ no seco, o que demonstra a contribuição das nuvens e umidade do ar. Mesmo sabendo que a temperatura do ar é maior no período seco, vemos que a Ra recuou $3 \%$ em relação ao período chuvoso justamente por conta da menor nebulosidade e umidade do ar, enquanto o Rs foi 456,46 W.m-2 (chuvoso) e 470,60 W.m² (seco), em média.

Como a Rs foi sempre maior que a $\mathrm{Ra}$, a irradiância líquida de onda longa (BOL) foi sempre negativa em ambas estações, mas com maior emissão por parte do dossel na estação seca devido às temperaturas mais altas, então o BOL aumentou $103,19 \%$ da estação chuvosa para seca $\left(-25,39 \mathrm{~W} \cdot \mathrm{m}^{-2}\right.$ para $-51,59$ W. $\left.m^{-2}\right)$.

\section{AGRADECIMENTOS}

Ao Conselho Nacional de Desenvolvimento Científico e Tecnológico - CNPq pelo apoio ao desenvolvimento e financiamento deste projeto. A Usina Coruripe Açúcar e Álcool S/A pelo apoio e por ter disponibilizado a área de estudo e nossa permanência em suas dependências.

\section{REFERÊNCIAS}

AGUIAR, L. J. G. Balanço de radiação em áreas de floresta e pastagem em Rondônia. Programa de Pós-graduação em Meteorologia Agrícola, Universidade Federal de Viçosa, Dissertação de Mestrado, p. 70, 2007.

BASTABLE, H. G.; SHUTtLeWORTH, W. J.; DALlAROSA, R. L. G.; FISCH, G.; NOBRE, C. A. Observations of climate albedo, and surface radiation over cleared and undisturbed Amazonian forest. $\begin{array}{llllllll}\text { International Journal of Climatology, } & \text { v. } 13, \quad \text { n. } & 7, & \text { p. } & 783-796, & \end{array}$ https://doi.org/10.1002/joc.3370130706

BIUDES, M. S.; VOURLITIS, G. L.; MACHADO, N. G.; ARRUDA, P. H. Z.; NEVES, G. A. R.; LOBO, F. A.; NEALE, C. M. U.; NOGUEIRA, J. S. Patterns of energy Exchange for tropical ecosystems across a climate gradiente in Mato Grosso, Brazil. Agricultural and Forest Meteorology, v. 202, p.112-124, 2015. https://doi.org/10.1016/i.agrformet.2014.12.008

CARNEIRO, R. G.; MOURA, M. A. L.; LYRA, R. F. F.; ANDRADE, A. M. D.; SANTOS, A.B.; MAIA, R. G. $X$. Fluxo de calor do solo e saldo de radiação dentro de uma área de Mata Atlântica em comparação com uma de cana-de-açúcar. Revista Brasileira de Geografia Física, v. 6, n.03, p. 555-565, 2013.

FERREIRA JUNIOR, R. A.; SOUZA, J. L.; ESCOBEDO, J. F.; TEODORO, I.; LYRA, G.B.; ARAÚJO NETO, R. A. Cana-de-açúcar com irrigação por gotejamento em dois espaçamentos entrelinhas de plantio. Revista Brasileira de Engenharia Agrícola e Ambiental, v. 18, n.8, p. 798-804, 2014. https://doi.org/10.1590/1807-1929/agriambi.v18n08p798-804

GALVÃO, J. A. C.; FISCH, G. Balanço de radiação em área de pastagem na Amazônia. Revista Brasileira de Agrometeorologia, v. 8, n. 1, p 1-10, 2000.

IQBAL, M. An introduction to solar radiation. Ed. AP. Vancouver, Canadá, p. 389, 1983.

LOPES JÚNIOR, J. M.; MOURA, M. A. L.; COSTA, N. B.; SANTOS, M. F.; VASCONCELOS, F. L. 2018. Frequência e probabilidade de ocorrência de transmitância atmosférica global para a região de MaceióAlagoas. In: CONGRESSO BRASILEIRO DE METEOROLOGIA, 20, 2018, Maceió. Anais... Maceió: recurso eletrônico, 2018, 43-57. 
LYRA, R.; NOBRE, C.; FISCH, G.; ROCHA, E.; ROCHA, H.; SOUZA, S. Efeitos do desmatamento sobre a termodinâmica da baixa atmosfera. In: CONGRESSO BRASILEIRO DE METEOROLOGIA, 8, 1994, Belo Horizonte. Anais. Belo Horizonte-MG: CBMET 1994, 81-84.

MACHADO, M. A. B. L. Florística e fitossociologia do estrato arbóreo de fragmentos de mata atlântica da usina Coruripe - estado de Alagoas. Programa de Pós-graduação em Agronomia, Universidade Federal de Alagoas, Dissertação de Mestrado, p. 100, 2003.

MALEK, E. Evaluation of effective atmospheric emissivity and parameterization of cloud at local scale. Atmospheric Research, v 45, p. 41-54, 1997. https://doi.org/10.1016/S0169-8095(97)00020-3

MMA - Ministério do Meio Ambiente. Mata Atlântica. 2010. Disponível em: $<$ http://www.mma.gov.br/biomas/mata-atlantica>. Acesso em: 16 de junho de 2018.

NOBRE, C. A.; SAMPAIO, G.; SALAZAR, L. Mudanças climáticas e Amazônia. Ciência e Cultura, v. 59, n. 3, p. 22-27, 2007.

OKE, T.R. Boundary Layer Climates. London, Methuen Co., p. 435, 1987.

PAI, E. D.; ESCOBEDO, J. F. Estimativa da radiação atmosférica em função dos índices radiométricos kt e kd para Botucatu-SP. Energia na Agricultura, Botucatu, v. 30, n. 2, p. 172-179, 2015. https://doi.org/10.17224/EnergAgric.2015v30n2p172-179

PASHIARDIS, S.; KALOGIROU, S. A.; PELENGARIS, A. Characteristics of longwave radiation through the statistical analysis of downward and upward longwave radiation and inter-comparison of two sites in Cyprus. Journal of Atmospheric and Solar-Terrestrial Physics, v. 164, p. 60-80, 2017. https://doi.org/10.1016/i.jastp.2017.08.007

PEZZOPANE, J. E. M.; REIS, G. G.; REIS, M. D. G. F.; DA COSTA, J. M. N.; CHAVES, J. H. Temperatura do solo no interior de um fragmento de floresta secundária semidecidual. Revista Brasileira de Agrometeorologia, v. 10, n. 1, p. 1-8, 2002.

PIRES, W. N.; MOURA, M. S. B.; AIDAR, S. T.; SOUZA, L. S. B. Temperatura foliar e do dossel como indicador de déficit hídrico em plantas da caatinga: resultados iniciais. In: SIMPÓSIO DE MUDANÇAS CLIMÁTICAS E DESERTIFICAÇÃO NO SEMIÁRIDO BRASILEIRO, 4, Petrolina, 2015. Experiências e oportunidades para o desenvolvimento. Anais... Petrolina: Embrapa Semiárido, 2015.

PRATA, A. J. A new long-wave formula for estimating downward clear - sky radiation at the surface. Quartely Journal of the Royal Meteorological Society, v. 122, p. 1127-1151, 1996. https://doi.org/10.1002/qj.49712253306

QUERINO, C. A. S.; MOURA, M. A. L.; QUERINO, J. K. A. S., VON RADOW, C.; MARQUES FILHO, A. D. $\mathrm{O}$. Estudo da radiação solar global e do índice de transmissividade (kt), externo e interno, em uma floresta de mangue em Alagoas-Brasil. Revista brasileira de Meteorologia, v. 26, n. 2, p. 204-294, 2011. https://doi.org/10.1590/S0102-77862011000200005

QUERINO, C. A. S. Estimativa da radiação de onda longa atmosférica horária no estado de Mato Grosso. Programa de Pós-graduação em Física Ambiental, Universidade Federal de Mato Grosso, Tese de Doutorado, p. 56, 2016.

QUERINO, C. A. S.; BIUDES, M. S.; MACHADO, N. G.; QUERINO, J. K. A. S.; SANTOS NETO; DA SILVA; A. P.; NOGUEIRA, J. S. BALANÇO DE ONDAS CURTAS SOBRE FLORESTA SAZONALMENTE ALAGÁVEL DO PANTANAL MATO-GROSSENSE. Revista Brasileira de Climatologia, v. 20, p. 252266, 2017. https://doi.org/10.5380/abclima.v20i0.50701

SANTOS, T. V. Aspectos da distribuição temporal da precipitação e suas relações com as brisas na cidade de Maceió - AL. Universidade Federal de Alagoas, TCC (trabalho de Conclusão de Curso), p. 51, 2006. 
SEMARH - Secretaria do Estado do Meio Ambiente e Recursos Hídricos de Alagoas. Boletim Informativo das condições climáticas do Estado de Alagoas. 2017. Disponível em: $<h t t p: / / w w w . s e m a r h . a l . g o v . b r />$. Acesso em: 28 de maio de 2018.

SINGH, N.; BHATTACHARYA, B. K.; NANDA, M. K.; SONI, P.; PARIHAR, J. S. Radiation and energy balance dynamics overyoung chir pine (Pinus roxburghii) system in Doon of western Himalayas. Journal of Earth System Science, v. 123, n. 7, p. 1451-1465, 2014. https://doi.org/10.1007/s12040-014-0480-6

SOS Mata Atlântica. INPE - Instituto Nacional de Pesquisas Espaciais. Atlas dos remanescentes florestais da mata atlântica. Relatório técnico, 2018.

SOUZA, J. L.; NICÁCIO, R. M.; MOURA, M.A.L. Global solar radiation measurements in Maceió, Brazil. Renewable Energy, v. 30, n. 8, p. 1203-1220, 2005. https://doi.org/10.1016/j.renene.2004.09.013

SOUZA-FILHO, J. D. C.; RIBEIRO, A.; COSTA, M. H.; COHEN, J. C. P.; ROCHA, E. J. P. Variação sazonal do balanço de radiação em uma floresta tropical no Nordeste da Amazônia. Revista brasileira de Meteorologia, v. 21, n. 3b, p. 318-330, 2006.

Recebido em: 22/10/2019

Aceito para publicação em: 28/01/2020 